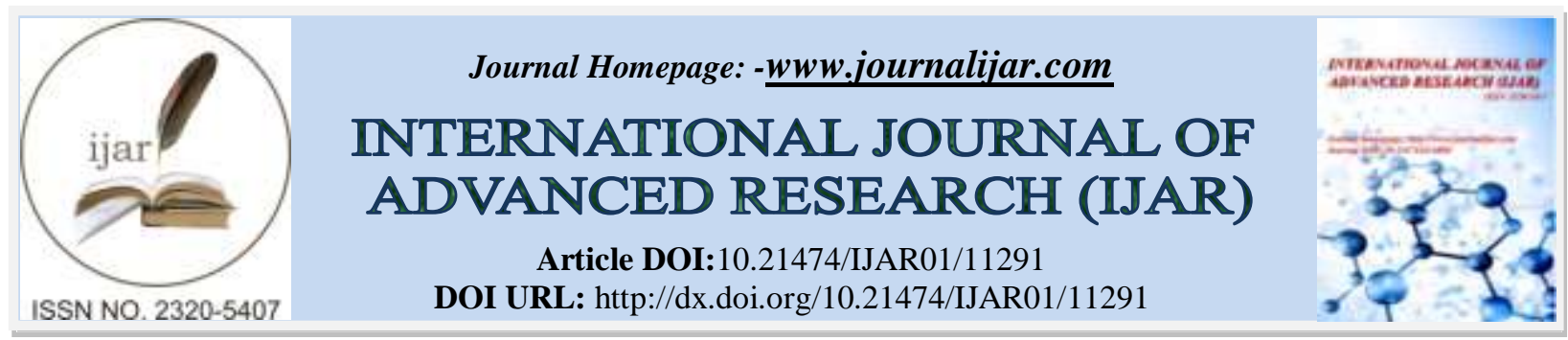

RESEARCH ARTICLE

\title{
A COMPREHENSIVE REVIEW OF BASIC IMPRESSION TECHNIQUES FOR IMPLANT RESTORATIONS
}

\author{
Dr. Aswati Soman ${ }^{1}$, Dr. Aby Mathew $\mathbf{T}^{2}$, Dr. Suja Joseph ${ }^{3}$, Dr. Annie Susan Thomas ${ }^{3}$, Dr. Minnu Harsha \\ Kumar $^{1}$ and Dr. Saranya Y.S ${ }^{1}$ \\ 1. PG Resident, Department of Prosthodontics, Pushpagiri College of Dental Sciences, Thiruvalla, India. \\ 2. Professor and HOD, Department of Prosthodontics, Pushpagiri College of Dental Sciences, Thiruvalla, India. \\ 3. Professor, Department of Prosthodontics, Pushpagiri College of Dental Sciences, Thiruvalla, India.
}

\section{Manuscript Info}

\section{Manuscript History}

Received: 05 May 2020

Final Accepted: 10 June 2020

Published: July 2020

Key words:-

Closed Tray Technique, Dental Implant,

Digital Impression, Impression

Techniques, Open Tray Technique

\begin{abstract}
Dental implant, a fixed prosthesis widely used to replace the missing natural tooth for the rehabilitation of both partially and fully edentulous patients. In modern dentistry, dental implant plays a vital role in restoring the form, function, and esthetics of the patients. Passive fit between the implant abutment interfaces is one of the most significant pre-requisites of implant - supported prosthesis. Although achieving an absolute passive fit is not yet possible, providing the best possible fit is fundamental to prevent future biologic and mechanical complications that include screw loosening and/or fracture that can lead to implant failure. An accurate impression relates the coronal structure of an implant to other structures of the oral cavity and avoiding instabilities in the prosthetic device and thereby provides long-term success. Here in this article, a brief overview of implant impression techniques is outlined.
\end{abstract}

Copy Right, IJAR, 2020,. All rights reserved.

\section{Introduction:-}

Dental Implantology is an exciting field of dentistry that is helping clinicians to restore patients with better functional and esthetic outcomes. The current generation of dental implants was first presented to the dental profession by Dr. P.I Branemark in 1977, but their use was restricted to the anterior edentulous mandible. ${ }^{[1]}$ But later on, the success of osseointegration became apparent, osseointegrated implants have been successfully used as an alternative over other conventional approaches in replacing the missing natural teeth. ${ }^{[2]}$ Dental implants widened the scope of clinical dentistry by providing treatment options in complex cases where rehabilitation was very difficult.

Fabricating an implant-supported fixed prosthesis is similar to fabricating a conventional fixed prosthesis. But in implant-supported prosthesis, it is very essential to record the three-dimensional position of the implants in patient's mouth. ${ }^{[3-4]}$ Natural teeth have a periodontal ligament to compensate minor inaccuracies in the positioning of the abutments. But the dental implants directly ankylosed with the bone, lacks the inherent mobility of the periodontal ligament. ${ }^{[3-5]}$ Hence they cannot accommodate distortion or misfit at a healthy bone-implant interface. ${ }^{[3-4]}$ Long term clinical success of implant-supported prosthesis depends on passive fit between the implant and the abutment. The discrepancies in the passive fit due to inaccurate impressions may lead to dental implant failure due to mechanical and/or biological complications. ${ }^{[3-7]}$

Corresponding Author:- Dr. Aswati Soman

Address:- PG Resident, Department of Prosthodontics, Pushpagiri College of Dental Sciences,

Thiruvalla,India. 
So when fabricating implant prosthesis, reproducing the accurate position of the implant in the definitive cast is essential. ${ }^{[3]}$ It should reproduce the correct 3-dimensional orientation rather than the surface details. The precision of the definitive cast is essential for the proper fit of the implant prosthesis, which depends upon an accurate impression. ${ }^{[3-7]}$ The accuracy of the impression is affected by the impression technique, impression coping position, implant angulations, number, and area of implant placement. ${ }^{[7]}$ Among these, the impression technique is considered as the major factor. Several impression techniques have been suggested to obtain a master cast that will ensure the passive fit of implant prosthesis. ${ }^{[8]}$.

Various Impression Techniques for Implant:

Basic impression techniques for the implant are

1. Implant level impression technique.

2. Abutment level impression technique.

\section{Implant Level Impression ${ }^{[4,9-12]}$ :}

A universal impression technique aids in the fabrication of any type of restoration. This impression provides more freedom for the selection and modification of an abutment. This technique is commonly applied when the implant is not ideally placed. There are 2 types of impression techniques

1. Open tray Impression techniques

2. Closed tray Impression techniques

Open tray Impression technique ${ }^{[4,9-12]}$ :

Open tray impression technique [Fig.1] ${ }^{[4]}$ also known as direct or pick up impression technique. In this technique, the impression coping used for impression making is removed from the mouth along with the set impression. Then implant analogs are attached to the impression copings while they are embedded in the impression tray. So a custom or stock open tray with window space corresponding to the implant site is required, to expose the impression screw through the impression made. This helps in loosening the screw and facilitate easy removal of the impression coping from patient's mouth.

When replacing a missing teeth in an entire arch or replacing multiple tooth using implants, the open tray technique is better than the closed tray technique with higher accuracy. ${ }^{[4]}$ This technique compensates the inaccuracy that can occur due to implant angulation, deformation of the impression material upon recovery from the mouth, and eliminates the error while replacing the impression copings into the impression. ${ }^{12]}$

Indications of open tray impression technique ${ }^{[10,12]}$ :

1. Multiple number of implants which are not parallel to each other.

2. Full arch implant supported fixed prosthesis.

3. The open tray technique is also helpful if the implants are seated very deep in the soft tissue, as the exposure of short closed tray coping through soft tissue is difficult.

Advantages of Open Tray ${ }^{[10,12]}$ :

1. The dentist can confirm the laboratory preparation and contour of the provisional prosthesis before final crown fabrication.

2. Reduces the effect of implant angulation.

3. Reduces the deformation of the impression material.

4. Eliminates the repositioning of the coping back into the impression after removal.

Disadvantages of Open Tray ${ }^{[10,12]}$ :

1. Blind attachment of the implant analog to the impression coping may result in a misfit of components.

2. Require a custom tray, or a modified prefabricated tray.

3. Need to find and expose the protruding screws of the impression posts before setting of the impression the material.

4. Some rotational movement of the impression coping may occur when securing the implant analog.

5. Difficulty to apply in the posterior regions due to the increased height of the screws.

6. Longer working time is needed intraorally, as all screws have to be loosened before removing the impression. 

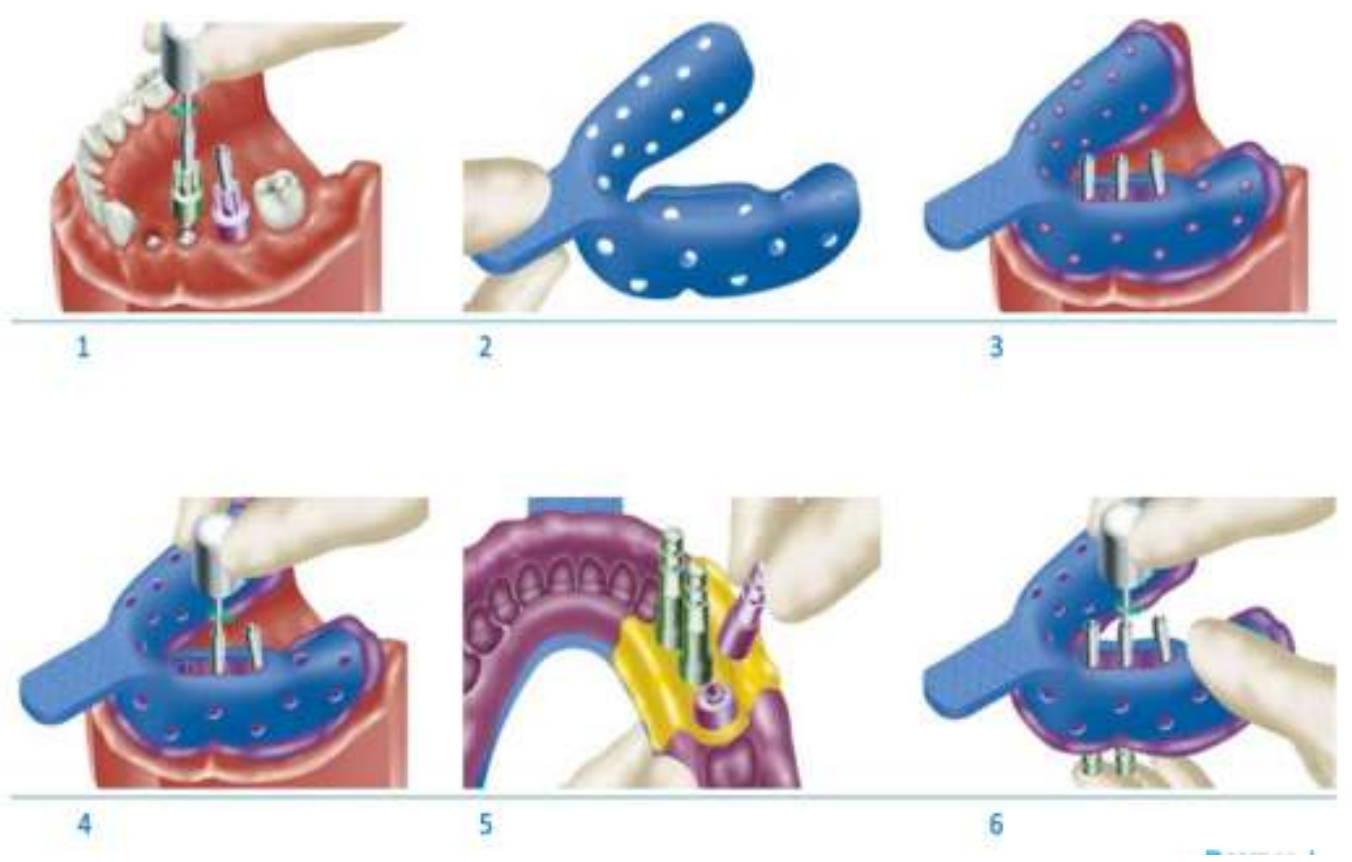

Fig 1:- Open tray impression technique.

\section{Splinted impression technique ${ }^{[4,10]}$ :}

In the splinted technique, a splinting material is used to connect all the impression coping before the impression is made. This technique is recommended in cases where multiple implants are used to replace missing teeth. Splinting of the transfer coping decreases the amount of distortion of the impression coping during removal and improves accuracy and implant stability and also helps to prevents movement of impression copings during analog fastening, which provides better results than not splinting. Accuracy of a splinted impression technique depends upon its resistance to deformation under the forces of impression material; hence the use of rigid splint material is essential for accurate master cast. Materials used to splint impression copings include pattern resin, light-curing composite resin, and impression plaster and autopolymerizing acrylic resin.

\section{Closed tray impression technique ${ }^{[4,9-12]}$ :}

Also known as indirect technique or transfer impression technique [Fig.2.] ${ }^{[4]}$ The coping used for this technique is called Closed tray impression coping. In this technique, the implant copings are connected to the implant, and the impression is made in a stock tray or custom tray. Once the impression is set it is removed, leaving the copings in the patient's mouth itself. Impression copings are then removed from the implant and attached to the implant analogs. Then this coping-analog assembly is repositioned manually into the impressions and cast is poured. While transferring the coping, make sure that the impression coping is inserted with the same orientation as in the mouth.

\section{Indications $^{[10,12]}$ :}

1. Limited inter arch space

2. Difficult access in the posterior region of the mouth

3. Tendency to gag

Advantages of Closed Tray ${ }^{[10,12]}$

1. Easier and less time consuming

2. Simple procedure, similar to normal impression techniques used for natural dentition.

3. Suitable for short inter arch distance.

4. Visual tightening of the analog to the coping is more accurate.

5. The use of a custom tray or a modified prefabricated tray is not necessary

Disadvantages of Closed Tray ${ }^{[10,12]}$

1. Replacing the coping back into its respective space in the impression may cause inaccuracy in recording the exact spatial position of implants in the oral cavity. 
2. Difficulty in impression removal can induce strain in the mass of impression material.

3. Difficulty in removing the impression if there is angulated implants.

4. Not suitable for deeply placed implants.

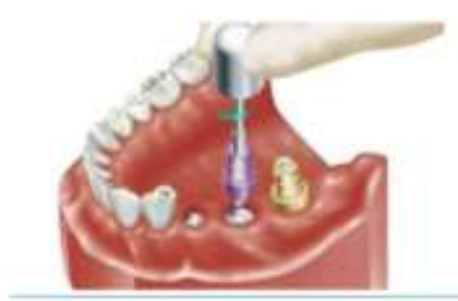

1

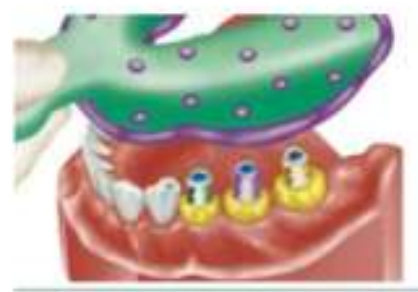

4

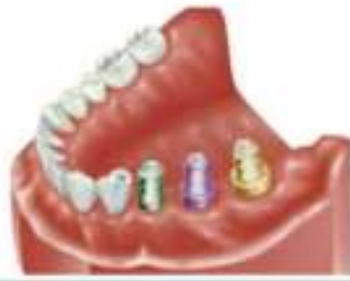

2

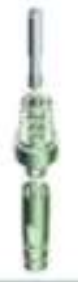

5

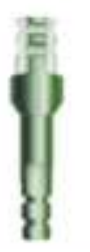

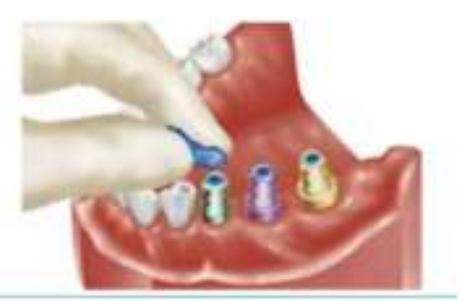

3

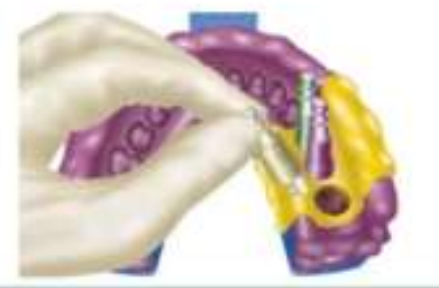

\section{6}

Fig 2:- Closed tray impression technique.

Abutment Level Impression ${ }^{[4,9-12]}$ :

An abutment level impression is indicated when there is a need to replace the existing old implant- supported crown. This procedure is similar to the normal crown and bridge technique. In this technique, the final abutment is fixed on top of the implant in the patient's mouth. Then the abutment is prepared with a diamond or carbide bur. These abutments are prepared directly in the patient's mouth to follow the gingival contour. Once the abutment is prepared, an impression is made using polyether or addition silicone material and poured with a high strength gypsum material [Fig.3]. ${ }^{[4]}$ Then prosthesis is fabricated in this model and cemented in the patient's mouth.

\section{Indications $^{[10,12]}$ :}

1. Single-body/one-piece implant.

2. Implant placement in the non-aesthetic posterior region.

3. Cement-retained prosthesis where high precision is not required.

Advantages of Abutment level impressions ${ }^{[10,12]}$

1. Simple provisional restoration fabrication

2. Selecting abutments in the laboratory

3. For custom-made abutments

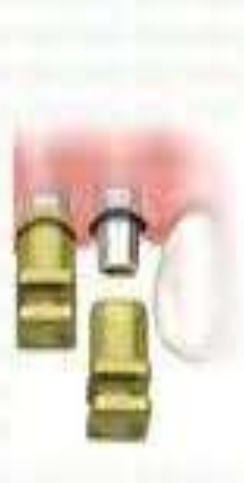

Abutmentlevel
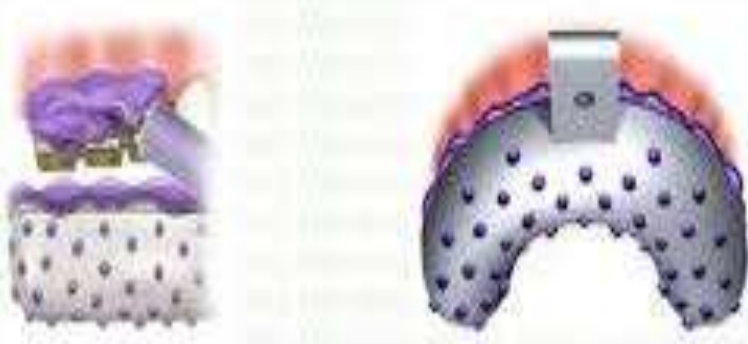

Fig. 3:- Abutment level implant impression. 
Snap-fit (press fit) plastic impression coping technique ${ }^{[10-12]}$

The International Team for Implantology (ITI) has introduced the Snap-On (press fit) impression technique. This technique uses press-fit impression coping which is connected to the implant by pressing instead of screwing the plastic impression copings are picked up in the impression [Fig.4]. ${ }^{[3]}$ This technique combines the benefits of both open tray and closed tray impression techniques. Although it is similar to an open tray technique, it does not require an open tray and eliminate the need for large tray holes and long guide screws that are difficult to place in mouths with a narrow opening. It is not a transfer impression, either, because the plastic impression copings are picked up in the impressions.

Advantages ${ }^{[10-12]}$

1. Reliable impression technique as it helps to overcome movement of coping inside the impression material

2. Can be used in both open and closed tray implant impression techniques

3. More comfortable for both the clinician and the patient

4. Easy to manipulate

5. Time- saving

\section{Disadvantages ${ }^{[10-12]}$}

Issues with attaching the implant analog to the impression coping or some errors due to distortion of plastic caps may affect the accuracy.

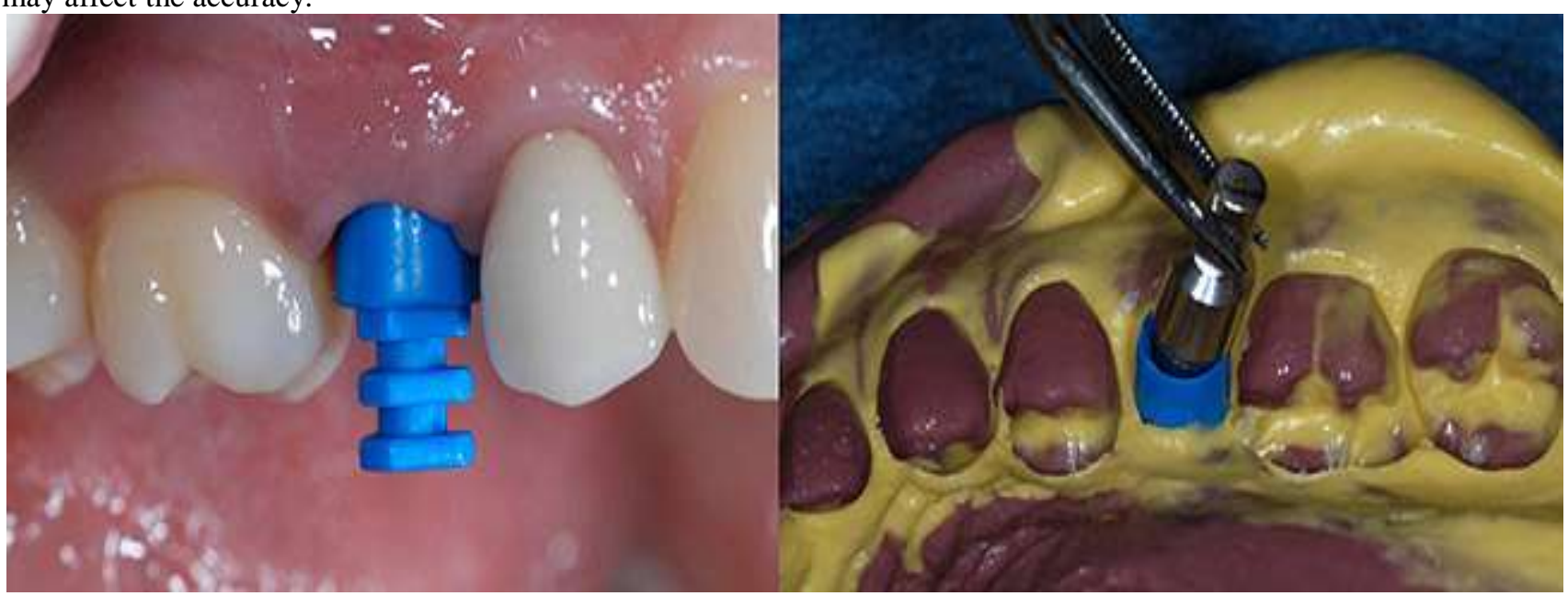

Fig 4:- Snap fit implant impression technique.

\section{Digital Impression Technique ${ }^{[4,9-12]}[\text { Fig.5 }]^{[4]}$}

Over the past few years, digital technologies have developed rapidly. And this breakthrough leads to the adoption of computer-aided design and computer-aided manufacturing (CAD/CAM) to construct implant prosthesis. This technology utilizes intraoral digital scanners which have revolutionizing the way we take impressions. The digital implant impression technique has become an effective alternative for the conventional impression technique. Taking a digital impression using an intraoral optical scanner (IOS) helps create a virtual model that uses the digital impression immediately, and eliminates the need for a stone model, which improves the accuracy and fit of the final prosthesis.

The main requirements for the CAD/CAM are ${ }^{[10]}$

1. The digital scanner, which scans and transforms the geometry into the digital data which can be processed by the computer

2. Software that processes the data and creates a $3 D$ virtual model via CAD

3. A production technology that converts the scanned data to produce a precise replica of CAD model

\section{Advantages ${ }^{[10]}$}

1. Reduced distortion by circumventing the use of dental materials such as impression and gypsum materials

2. Eliminates the need for disinfection and shipping of impression to the laboratory

3. Simple device disinfection required 
4. Improved patient experience and comfort during impression making

5. Digital assessment of available space for the implant prosthesis, depth of implant placement and emergency profile configuration can be done before proceeding with laboratory steps

6. Easy to store the scanned data for future references.

7. More efficient and predictable

8. Chair side assessment of implant position can be done accurately

9. Possibility to rescan the poorly captured areas without repeating the entire impression

10. Easy to communicate with the patient and technician.

\section{Disadvantages ${ }^{[10]}$}

1. A considerable financial investment is required to purchase an intraoral scanner.

2. Inability to scan the proximal area of the neighbouring tooth when situate too close to the abutment

3. Crucial factor in case of digital impression is to apply the right intraoral scanning protocol and to accept the learning curve.

4. In case of multiple implants difficult to identify the position of the abutments.

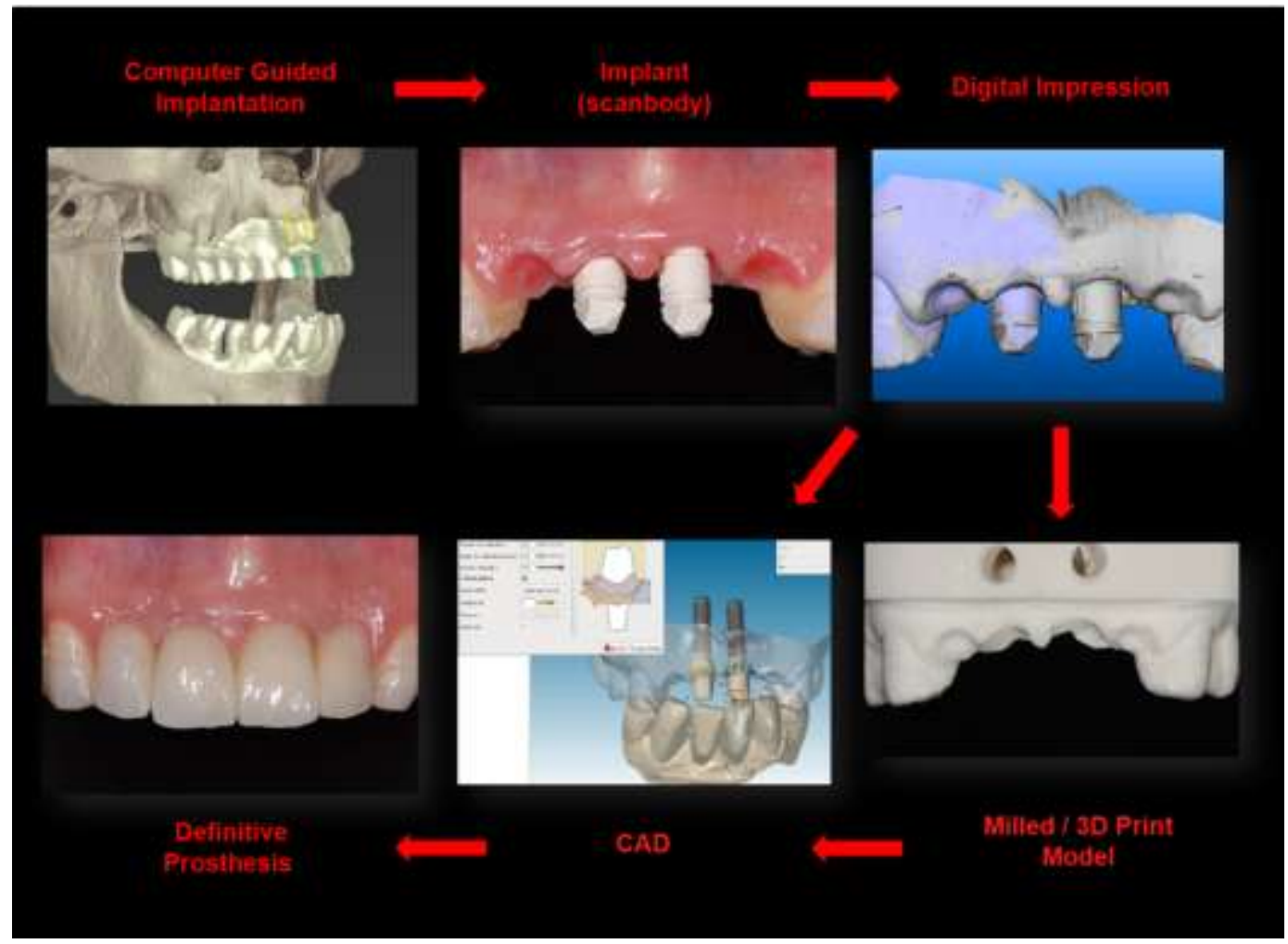

Fig 5:- Digital impression.

\section{Conclusion:-}

Accurate impressions and meticulous attention in recording complete details provide a foundation for long term durability and success of the prosthesis in implant prosthodontics. Comprehensive understanding of the wide range of basic prosthetic components, implant impression materials, implants impression techniques will provide paramount skills in performing clinical procedures in implant dentistry. Impression techniques in particular either open or closed tray techniques have their respective merits and drawbacks and the choice of technique employed can be down to clinician preference. And this article gives a brief idea about different impression techniques, its advantages and drawbacks.

\section{References:-}

1. Becker CM, Kaiser DA, Jones JD. Guidelines for splinting implants. The Journal of prosthetic dentistry. 2000 Aug 1;84(2):210-4. 
2. Deogade SC. An alternative procedure of splinting multiunit implant copings to minimize the resin shrinkage. Journal of Dental Implants. 2015 Jul 1;5(2):124.

3. Rutkunas V, Ignatovic J. A technique to splint and verify the accuracy of implant impression copings with lightpolymerizing acrylic resin. The Journal of prosthetic dentistry. 2014 Mar 1;111(3):254-6.

4. Kalpana D, Smitha Sharan D, Sreeharsha TV, Pradeep Chandra K, Brunda K, Nadira Jabeen S. A review on dental implant impressions. International Journal of Applied Dental Sciences 2019 Jan; 5(1): 33-36

5. Shankar YR, Sahoo S, Krishna MH, Kumar PS, Kumar TS, Narula S. Accuracy of implant impressions using various impression techniques and impression materials. Journal of Dental Implants. 2016 Jan 1;6(1):29.

6. Carr AB. Comparison of impression techniques for a five- implant mandibular model. Int J Oral Maxillofac Implants 1991;6:448- 55.

7. Tafti AF, Hatami M, Razavi F, Ebadian B. Comparison of the accuracy of open-tray and snap-on impression techniques of implants with different angulations. Dental Research Journal. 2019 Nov;16(6):413.

8. Revilla-León M, Sánchez-Rubio JL, Oteo-Calatayud J, Ozcan M. Impression technique for a complete-arch prosthesis with multiple implants using additive manufacturing technologies. The Journal of prosthetic dentistry. 2017 Jun 1;117(6):714-20.

9. Pandey KK, Sahu M, Tiwari AK, Shrestha S, Gaur A, Ahmad N. A Beginners Guide for Implant Impression: Review Article. Int J Oral Health Med Res 2017;4(1):91-93.

10. Brainerd SS. Impression Techniques in Implant Dentistry. IOSR Journal of Dental and Medical Sciences 2018 Nov;17(11):33-44.

11. I Gayathridevi SK, Gowda H, Vaishali K. Impression techniques in implants. Journal of Dental and Orofacial Research. 2016 Aug;12(2):11-19.

12. Kalamalla A, Haripriya P, Perisetty D, Rasool M, P Lakshmaiaha, Pavankumar Y. A Comprehensive Review of Basic Implant Impression Techniques. EC Dental Science. 2020 Mar;SI.02: 01-11.

13. Kurtzman GM. Creating Great Dental Impressions.inside dental assisting 2010jan/feb 8(1). 